\title{
Structural and magnetic roughness in a Co/Ru multilayer patterned into a large scale hexagonal array
}

\author{
C. J. Kinane \\ School of Physics and Astronomy, E. C. Stoner Laboratory, University of Leeds, Leeds, LS2 9JT, \\ UK and ISIS, Rutherford Appleton Laboratory, Chilton, Didcot, OX11 OQX, UK \\ N. A. Porter, C. H. Marrows, ${ }^{\text {a) }}$ and B. J. Hickey \\ School of Physics and Astronomy, E. C. Stoner Laboratory, University of Leeds, Leeds, LS2 9JT, \\ $U K$ \\ D. A. Arena \\ National Synchrotron Light Source, Brookhaven National Laboratory, Upton, New York 11973-5000, USA \\ J. Dvorak \\ Department of Physics, Montana State University, Bozeman, Montana 59717, USA \\ E. Sirotkin and F. Y. Ogrin \\ School of Physics, University of Exeter, Stocker Road, Exeter EX4 4QL, UK \\ T. Charlton and S. Langridge \\ ISIS, Rutherford Appleton Laboratory, Chilton, Didcot, OX11 OQX, UK
}

(Presented on 7 November 2007; received 11 September 2007; accepted 3 October 2007; published online 11 February 2008)

Self-assembled arrays of nanospheres have been used to pattern a $\mathrm{Co} / \mathrm{Pt}$ multilayer into nanopillars. On top of this has been deposited a Co/Ru multilayer, which is antiferromagnetically coupled. The nanopillars introduce a known structural and magnetic lateral modulation into the multilayer. Soft x-ray magnetic scattering was used to observe the interference patterned from the patterned substrate. This has allowed us to show how the magnetic roughness correlates with the structural roughness and obtain selective magnetometry of the various magnetic elements. (C) 2008 American Institute of Physics. [DOI: 10.1063/1.2829394]

There are great difficulties in obtaining information on buried interfaces in multilayer nanostructures. This is a crucial problem in magnetic information storage and spintronics research, where the magnetic properties are strongly coupled to the structure. Internal characterization of nanostructures is possible using neutron and $\mathrm{x}$-ray scattering, particularly offspecular scattering techniques to investigate lateral disorder. ${ }^{1,2}$ There has been great interest in lateral structures recently partly because the behavior of such systems, when patterned into large scale arrays, provides model systems that can be utilized to study scientifically interesting areas such as digital computation ${ }^{3}$ and artificial frustrated magnetism, ${ }^{4}$ as well as applications where surface magnetism is important like giant magnetoresistance (GMR) and tunneling magnetoresistance (TMR) devices and patterned media. ${ }^{5}$ In this case, large scale arrays are an ideal system to study the effects of small local deviations in the magnetic structure. These are of interest since these magnetic deviations can affect properties such as polarized electron transport. Scattering techniques allow averaged statistical quantities to be measured from arrays of devices more easily than from local probes on single devices. Past studies have concentrated on patterned samples with nonmagnetic structural modulations using both polarized neutron reflectivity and soft X-ray resonant magnetic scattering (SXRMS). ${ }^{6,7}$ We have made use of

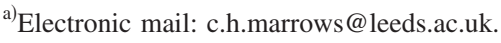

off-specular SXRMS in a previous study on structurally flat Permalloy, which has a domain pattern imprinted upon it by a perpendicularly magnetized $\mathrm{Co} / \mathrm{Pt}$ multilayer. ${ }^{8}$ In this report, self-assembled arrays of nanospheres were used to pattern perpendicularly a magnetized $\mathrm{Co} / \mathrm{Pt}$ multilayer into nanopillars. On top of this has been deposited a $\mathrm{Co} / \mathrm{Ru}$ multilayer, which is antiferromagnetically coupled, ${ }^{9}$ introducing a known structural and magnetic modulation.

The samples were prepared using a dc magnetron sputtering system with a base pressure of $\sim 5 \times 10^{-8}$ Torr. A partial pressure of 2.8 mTorr of Ar was used during deposition. The nominal structure of the samples was $[\mathrm{Co}(5 \AA) / \operatorname{Pt}(10 \AA)]$ $\times 20($ patterned $) / \operatorname{Ru}(400 \AA) /[\operatorname{Co}(31 \AA) / \operatorname{Ru}(31 \AA)] \times 20$, shown in the inset of Fig. 1. Co/Pt was deposited to obtain a perpendicularly magnetized film. ${ }^{10,11}$ This $\mathrm{Co} / \mathrm{Pt}$ multilayer was then patterned into a hexagonal dot array using commercially available suspensions of monodisperse polystyrene nanospheres with diameters of approximately $780 \mathrm{~nm}$. These formed a hexagonal template via self-assembly, allowing arrays with ordering over the range of $\sim 1 \mathrm{~cm}^{2}$. A combination of reactive ion etching and Ar ion milling was used to produce nanosphere capped $\mathrm{Co} / \mathrm{Pt}$ pillars. The spheres were then removed via abrasion. Further details on this process can be found in the following by Weekes et al. ${ }^{12,13}$ A scanning electron microscope (SEM) image of the patterned $\mathrm{Co} / \mathrm{Pt}$ multilayer is shown in Fig. 1. To ensure that there was 


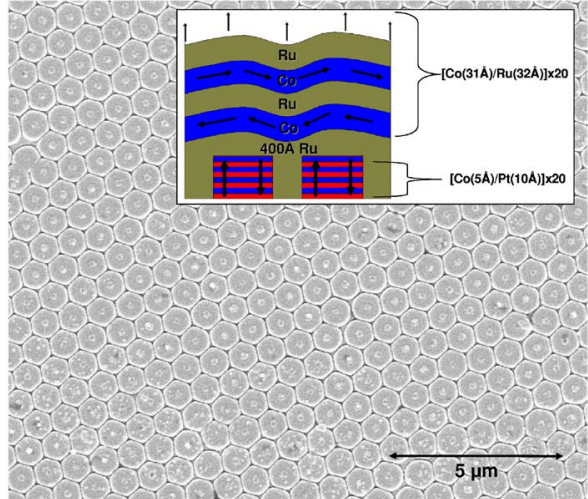

FIG. 1. (Color online) $16 \times 16 \mu \mathrm{m}^{2} \mathrm{SEM}$ image of $\mathrm{Co} / \mathrm{Pt}$ dots after milling. Inset: schematic of the complete sample structure.

no form of coupling other than magnetostatic between the $\mathrm{Co} / \mathrm{Pt}$ pillars and the following $[\mathrm{Co}(31 \AA) / \mathrm{Ru}(32 \AA)] \times 20$ multilayer, $400 \AA$ of $\mathrm{Ru}$ was then deposited to form a large nonmagnetic spacer layer. The $\mathrm{Co} / \mathrm{Ru}$ multilayer had thicknesses tailored to be on the third antiferromagnetically (AF) coupling peak. ${ }^{9}$

Figure 2(a) shows a $\mathrm{Cu} K \alpha$ rocking curve taken through the first order $\mathrm{Co} / \mathrm{Pt}$ Bragg peak for just the patterned $\mathrm{Co} / \mathrm{Pt}$ multilayer. The in-plane structure is clearly evident from the satellite peaks about $Q_{x}=0$. Vibrating sample magnetometry (VSM) loops are shown in Figs. 3(a) and 3(b) for the completed sample structure. Panel (a) shows the full VSM loop, while panel (b) shows a minor loop in the range of $\pm 400 \mathrm{Oe}$. VSM is sensitive to the bulk magnetization of the sample. However, the $\mathrm{Co} / \mathrm{Ru}$ moment is four times the size of the $\mathrm{Co} / \mathrm{Pt}$ moment, so it is evident that the majority of the minor loops in panel (b) comes from the $\mathrm{Co} / \mathrm{Ru}$.

In order to perform the SXRMS measurements, the samples were taken to the U4B beamline at the National Synchrotron Light Source. ${ }^{14} \mathrm{U} 4 \mathrm{~B}$ is equipped with an electromagnet able to apply a field of up to \pm 300 Oe in both the plane of the sample and the scattering plane. We define the coordinate system for scattering such that the $z$ direction lies normal to the sample, while the $x$ direction lies in both the sample and scattering planes. The sample geometry is such

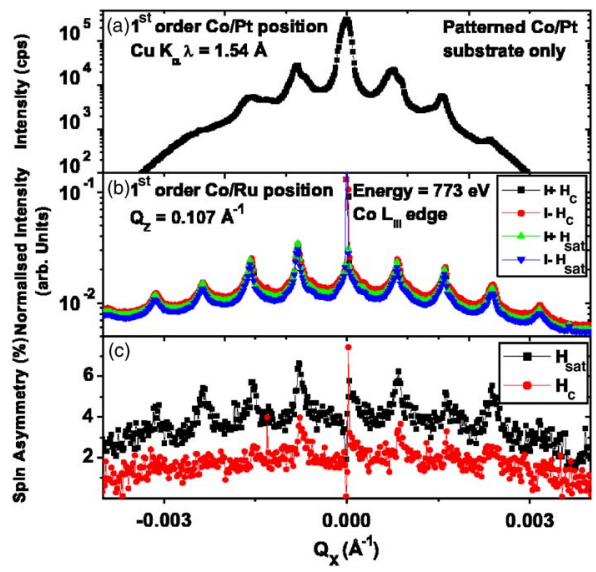

FIG. 2. (Color online) (a) $\mathrm{Cu} K \alpha \mathrm{x}$ rays on the $\mathrm{Co} / \mathrm{Pt}$ patterned substrate. (b) $Q_{x}$ scans at the first order $\mathrm{Co} / \mathrm{Ru}$ Bragg position $\left(Q_{z}=0.108 \AA^{-1}\right)$ at saturation and coercivity. (c) Spin asymmetry at saturation and coercivity.

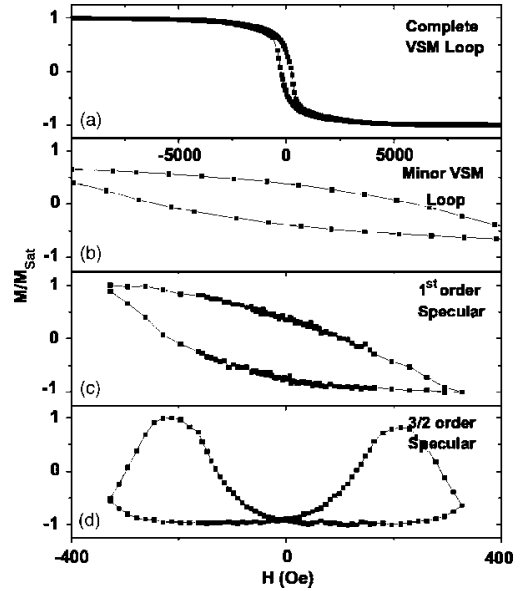

FIG. 3. (a) Full in-plane VSM loop. (b) Minor in-plane VSM loop. (c) SXRMS hysteresis loops at the first order specular position. (d) SXRMS hysteresis loops at the $3 / 2$ order specular position.

that the sample and detector angles allow the components of the elastic wavevector transfer $\mathbf{Q}$ to be selected in $x$ and $z$. X rays with a polarization of $90 \%$ were used, with the reflected intensity being normalized via a Au grid monitor upstream of the entrance slits to the scattering chamber.

In accordance with earlier studies ${ }^{15,16}$ at each point in reciprocal space, the scattered intensity was measured for both field directions $\left(I^{+}\right.$and $\left.I^{-}\right)$. The sum $\left(I^{+}+I^{-}\right)$and difference $\left(I^{+}-I^{-}\right)$are calculated, with the sum representative of the structure and magnetism self-correlations, and the difference the cross-correlations between the two. ${ }^{17} \mathrm{We}$ refer to the ratio $\left(I^{+}-I^{-}\right) /\left(I^{+}+I^{-}\right)$as the spin asymmetry (SA).

Energy scans (not shown) were recorded across the Co absorption edges, with the maximum in the SA being found close to the $L_{\mathrm{III}}$ edge at $772.5 \mathrm{eV}$ and the slight shift from the tabulated value of $778.1 \mathrm{eV}$ (Ref. 18) being due to our reflection geometry. A specular scan (not shown) was taken to determine the position of the $\mathrm{Co} / \mathrm{Ru}$ Bragg peak at $Q_{z}$ $=0.107 \AA^{-1}$. No $1 / 2$ order AF peak was observed in the specular scatter, which is in keeping with other studies using SXRMS. ${ }^{19}$ In order to probe the lateral magnetic structure, transverse $Q_{x}$ scans were taken at the $1 / 2$ order $\left(Q_{z}\right.$ $\left.=0.054 \AA^{-1}\right)$ and first order $\left(Q_{z}=0.107 \AA^{-1}\right)$ Bragg positions.

Figure 2(b) shows the $Q_{x}$ scan at the first order Bragg peak for applied fields corresponding to saturation and coercivity. The positions of the in-plane Bragg peaks are in excellent agreement with the $\mathrm{Cu} K \alpha$ x-ray data shown in Fig. 2(a). The satellite peak positions are also in agreement with what would be expected for their positions via $Q_{x} \approx 2 \pi / d$, which for a center to center nanosphere separation gives a spacing in $Q_{x}$ of $\approx 8 \times 10^{-4} \AA^{-1}$. It is also clear that the $Q_{x}$ scans at coercivity are slightly more intense than the $Q_{x}$ scans at saturation due to there being more lateral ferromagnetic $(\mathrm{F})$ disorder at coercivity. There is a good agreement between both the $I^{+}$and $I^{-}$curves at both saturation and coercivity, indicating that the in-plane $\mathrm{F}$ domain disorder is strongly correlated to the structural modulation. This becomes more evident when looking at the SA shown in Fig. 2(c). The SA shows that at saturation, there is a definite magnetic correlation with the structural modulation. At coer- 
civity, any correlations of the ferromagnetic domains and the structure are very weak as there is no net magnetization, but peaks are still just about visible, probably due to the fact that the sample was not quite at coercivity during the measurements. $Q_{x}$ scans were also taken at the $1 / 2$ order position (not shown) in order to be sensitive to the nominal lateral AF disorder between the Co layers in the $\mathrm{Co} / \mathrm{Ru}$ multilayer. The curves displayed the same general features and behavior as the first order $Q_{x}$ scan, indicating some crossover between the AF and F order parameters in both cases. A Gaussian fit to the left hand side in-plane Bragg peak gives a coherence length of $\approx 5000 \mathrm{~nm}$ for the dots, comparing well the SEM data, while a fit to the diffuse background (with in-plane features removed) gives an in-plane random roughness of $\approx 420 \mathrm{~nm}$, which is of the same order as the size of the $\mathrm{Co} / \mathrm{Pt}$ dots upon which the $\mathrm{Co} / \mathrm{Ru}$ was deposited.

An advantage of the SXRMS technique is that by measuring the intensity variation as a function of applied field, it is possible to measure hysteresis loops. ${ }^{2,20}$ Loops were taken at both the first order and $3 / 2$ order specular positions in $Q_{z}$ and are shown in Fig. 3 along with a VSM measurement over the same field range for comparison. The first $\mathrm{Co} / \mathrm{Pt}$ feature is to be found at $Q_{z}=0.42 \AA^{-1}$; hence, the hysteresis measurements are sensitive to the $\mathrm{Co} / \mathrm{Ru}$ multilayer stack only.

The first order specular loop compares well to the corresponding minor VSM loop, implying that it is largely the macroscopic magnetization of the structure that is being probed. Hysteresis loops were also taken at the positions of the first trough- and in-plane bragg peaks in $Q_{x}$ (not shown) as these off specular loops are sensitive to in-plane structures such as domains. ${ }^{2}$ These were found to be of the same shape, indicating that the magnetic disorder is similar over a range of length scales as far out as $15 \mu \mathrm{m}$. The loop taken at the $3 / 2$ position in $Q_{z}$ is sensitive to the AF magnetic structure between the $\mathrm{Co}$ layers in the $\mathrm{Co} / \mathrm{Ru}$ multilayer. The unconventional loop shape is due to the intensity peaking at the coercive fields of the $\mathrm{Co} / \mathrm{Ru}$, where the greatest amount of AF order is present. This then dies off as the AF order is swept out of the sample. These loops are similar to the loops observed in GMR measurements, which are known to be sensitive to the AF order.

Magnetic roughness can have multiple forms such as lateral domain patterns, a nonuniform height distribution of the moments, and vectorial differences in the directions to which the moments point. It has been shown by Kinane et $a l^{8}$ that it is possible to measure the domain disorder only, with minimal contributions from the chemical disorder, and that the structure and magnetism are discernible from each other using SXRMS. Langridge et $a l^{6}$. have shown that the domain disorder does not necessarily correlate with the structural disorder in the active multilayer, while the magnetic roughness is driven by the structural feature size. In this case, we pattern both the structural modulation and the domain modulation. It is clear that the magnetic roughness locks in on the structure at saturation, while the domain disorder has little influence since it is locked to the domain structure of the dots below the active $\mathrm{Co} / \mathrm{Ru}$ multilayer. This is backed up by the SXRMS hysteresis loops, which do not vary in shape as a function of $Q_{x}$, which probe the different length scales. It seems a reasonable that different forms of magnetic roughness interact with the structure to different extents.

In conclusion, the magnetic roughness has been shown to follow the structural modulation at saturation, with little sensitivity to the magnetism at coercivity due to the low net magnetization. It is possible to obtain a variety of hysteresis loops as a function of position in reciprocal space. By selecting the correct position in reciprocal space, it is possible to select which part of the sample is measured. In this case, the $\mathrm{Co} / \mathrm{Ru}$ multilayer rather than the $\mathrm{Co} / \mathrm{Pt}$ dots, while moving into the diffuse scatter, allows different lateral length scales to be probed. We saw that the magnetic disorder was similar over a range of length scales as far out as $15 \mu \mathrm{m}$.

The authors thank Brookhaven National Laboratory for NSLS beamtime. We are grateful to EPSRC, and the STFC Centre for Materials Physics and Chemistry for funding. We acknowledge Lara San Emeterio Alvarez for the SEM image.

${ }^{1}$ S. Langridge, J. Schmalian, C. H. Marrows, D. T. Dekadjevi, and B. J. Hickey, Phys. Rev. Lett. 85, 4964 (2000).

${ }^{2}$ C. H. Marrows, P. Steadman, A. C. Hampson, L.-A. Michez, B. J. Hickey, N. D. Telling, D. A. Dvorak, and S. Langridge, Phys. Rev. B 72, 024421 (2004).

${ }^{3}$ J. I. Martin, J. Nogués, K. Liu, J. L. Vicentand, and I. K. Schuller, J. Magn. Magn. Mater. 256, 449 (2003).

${ }^{4}$ A. Imre, G. Csaba, L. Ji, A. Orlov, G. Bernstein, and W. Porod, Science 311, 205 (2006).

${ }^{5}$ R. F. Wang, C. Nisoli, R. S. Freitas, J. Li, W. McConville, B. J. Cooley, M. S. Lund, N. Samarth, C. Leighton, V. H. Crespi, and P. Schiffer, Nature (London) 439, 303 (2006).

${ }^{6}$ S. Langridge, L. A. Michez, M. Ali, C. H. Marrows, B. J. Hickey, T. R. Charlton, R. M. Dalgliesh, M. Toohey, E. W. Hill, S. McFadzean, and J. N. Chapman, Phys. Rev. B 74, 014417 (2006).

${ }^{7}$ L.-A. Michez, C. H. Marrows, P. Steadman, B. J. Hickey, D. A. Arena, H.-L. Zhang, D. G. Bucknall, and S. Langridge, Appl. Phys. Lett. 86, 112502 (2005).

${ }^{8}$ C. J. Kinane, A. K. Suszka, C. H. Marrows, B. J. Hickey, D. A. Arena, J. Dvorak, T. R. Charlton, and S. Langridge, Appl. Phys. Lett. 89, 092507 (2006).

${ }^{9}$ S. S. P. Parkin, N. More, and K. P. Roche, Phys. Rev. Lett. 64, 2304 (1990).

${ }^{10}$ L. S. E. Alvarez, G. Burnell, C. H. Marrows, K.-Y. Wang, A. M. Blackburn, and D. A. Williams, J. Appl. Phys. 101, 09F508 (2007).

${ }^{11}$ S. Landis, B. Rodmacq, and B. Dieny, Phys. Rev. B 62, 12271 (2000).

${ }^{12}$ S. M. Weekes, F. Y. Ogrin, and W. A. Murray, Langmuir 20, 11208 (2004).

${ }^{13}$ S. M. Weekes, F. Y. Ogrin, W. A. Murray, and P. S. Keatley, Langmuir 23, 1057 (2007).

${ }^{14}$ See http://www.nsls.bnl.gov/beamlines/beamline.asp?bliduu4b for more information.

${ }^{15}$ J. F. MacKay, C. Teichert, D. E. Savage, and M. G. Lagally, Phys. Rev. Lett. 77, 3925 (1996).

${ }^{16}$ J. W. Freeland, K. Bussmann, P. Lubitz, Y. U. Idzerda, and C.-C. Kao, Appl. Phys. Lett. 73, 2206 (1998).

${ }^{17}$ R. M. Osgood III, S. K. Sinha, J. W. Freeland, Y. U. Idzerda, and S. D. Bader, J. Appl. Phys. 85, 4619 (1999).

${ }^{18}$ A. Thompson, D. Attwood, E. Gullikson, M. Howells, K. Kim, J. Kirz, J. Kortright, I. Lindau, P. Pianetta, A. Robinson, J. Scofield, J. Underwood, D. Vaughan, G. Williams, and H. Winick, X-Ray Data Booklet (University of California, Berkeley, CA, 2001).

${ }^{19}$ T. P. A. Hase, J. D. R. Buchanan, B. K. Tanner, S. Langridge, R. M. Dalgliesh, S. Foster, C. H. Marrows, and B. J. Hickey, J. Appl. Phys. 93, 6510 (2003).

${ }^{20}$ C. Spezzani, P. Torelli, M. Sacchi, R. Delaunay, C. F. Hague, V. Cros, and F. Petroff, Appl. Phys. Lett. 81, 3425 (2002). 